\title{
Recycled Polymer Materials as Aggregates for Concrete and Blocks
}

\author{
Md. Mostafizur Rahman ${ }^{1 *}$, Md. Akhtarul Islam¹, Mainuddin Ahmed² and Md. Abdus Salam² \\ ${ }^{1}$ Department of Chemical Engineering and Polymer Science, \\ Shahjalal University of Science and Technology (SUST), Sylhet- 3114, Bangladesh \\ ${ }^{2}$ Housing and Building Research Institute, Darus-salam Road, Mirpur, Dhaka-1216 \\ Phone: 02-8060989, Fax: 02-8060773, E-mail:mostafizur_cep@yahoo.com
}

\begin{abstract}
The potential of recycled waste polymeric materials as a substitute for aggregates in concrete has been investigated in the study. Two different types of waste polymer, namely polyurethane formaldehyde (PUF) based packaging waste and high density polyethylene (HDPE) were recycled and used in the experiment. Concrete and masonry poly block specimens were prepared using recycled polymer materials, and test specimens were characterized. The effects of waste polymer materials on the mechanical, physical and morphological properties of concrete and poly blocks have been investigated. The result shows that the inclusion of waste polymer materials decreases density, porosity and water absorption of concrete and poly blocks significantly. Polyurethane formaldehyde (PUF) based block exhibited lower density than that of HDPE-based concrete. Due to exceptionally low density, recycled polymer modified blocks and concrete can be used in non-load bearing structures, floating structures and where light weight materials recommended.
\end{abstract}

Keywords: Waste Polymer, recycling, concrete, masonry poly block, porosity

\section{Introduction}

The rapid urbanization and industrialization all over the world has resulted in large deposition of waste polymer materials. The world's annual consumption of plastic materials has increased from around 5 million tons in the 1950 s to nearly 100 million tons in $2001^{1}$. The annual plastic consumption in the United States is more than 11 million tons and $80 \%$ of the post-consumer plastics are sent to landfills, $8 \%$ is incinerated and only $7 \%$ is recycled while, in UK, the amount of plastic waste generated was about 3 million tons in 2001 of which, only $7 \%$ was recycled.

In the Dhaka city corporation area, about 3,315 tons of solid waste had been generated per day during 2005, of which $4.15 \%$ was composed of plastic materials ${ }^{2}$. As such, 50,214 tons of plastic waste was disposed in the city at the rate of 137.57 tons/day. The report indicates that plastic waste generation increases at the rate of $10.43 \%$ per year in the amount of plastic waste.

Plastic waste materials consist of surplus, obsolete, broken, old plastic furniture, different household plastic materials, equipments, anti-static packaging materials and devices made of plastic. These polymer wastes are almost non-degradable in the natural environment even after a long period of exposure. In Bangladesh, however, some amount of polymer wastes have been recycled to use for making toys but huge amount of polymer wastes are still abandoned and not recycled, which causes serious problems such as clogging in drainage system, land infertility, wastage of resources and environmental pollution. Slow degradation in the natural environment may be an undesired property of this waste material from environmental view point, but

*Corresponding Author: Md. Mostafizur Rahman

E-mail: mostafizur cep@yahoo.com it may appear to be valuable property as construction material. Inclusion of polymer waste in concrete can be a proper utilization of this valuable property. Thus, utilization of waste polymer material in making concrete/mortar can be good solution to this environmental hazard. Several investigations have been carried out on the use of recycled waste polymer materials in concrete.

Rebeiz et al. ${ }^{3}$ investigated on the structural behaviors of polymer concrete beam using recycled plastic. AlManaseer and Dalal ${ }^{4}$ studied the effect of plastic aggregates on the bulk density and compressive strength of concrete. Soroushian et al. ${ }^{5}$ also reported the effect of plastic aggregates on compressive strength, Elzafraney et al. ${ }^{6}$ studied on energy-efficiency of buildings using recycled plastic aggregates in concrete. Kumar and Prakash ${ }^{7}$ characterized waste plastics and optimized modifier content (waste plastic). Ruzaidi et al. $^{8}$ prepared light weight concrete replacing conventional aggregate made of crushed stone with polystyrene waste aggregate. Batayneh et al. ${ }^{9}$ recommended recycled waste plastic materials modified concrete in certain civil engineering applications, Kumar et al. ${ }^{10}$ reported the effect of waste plastics on mechanical strength. Siddique et al. ${ }^{11}$ investigated on the effect of recycled plastic on the properties of fresh and hardened concrete.

Most of the articles found in the literature deal with recycling of HDPE, low density polyethylene (LDPE), Polypropylene (PP) etc. But very few information is available regarding recycling of polyurethane formaldehyde (PUF) -based polymer wastes and its use as construction materials. In view of this, HDPE- and PUF-based polymer materials have been recycled and 
used as aggregates partially replacing conventional aggregates in the preparation of concrete and poly blocks, and the effect of waste polymer materials on the mechanical and physical properties of concrete/poly blocks have been investigated in this study. The upper limit of the recycled waste polymer in the mouldcomposition has also been determined.

\section{Experimental}

Specification of materials used in the study, recycling method of waste polymer, and test sample preparation has been discussed in the section.

\subsection{Materials}

The components of the concretes in this study were Portland cement, sand (fine aggregate) and coarse aggregates (stone chips and recycled waste plastic materials), while poly blocks were comprised of Portland cement, sand and PUF. Portland cement, stone chips and sand used in the experiment were collected from localities, and the waste plastics (HDPE and PUFbased polymer materials) used in this study had been procured from waste plastic wholesaler. Some of the basic properties of HDPE and PUF are presented in the Table 1 . The particle size of the sand used as fine aggregate was in the range of $0.15-4.75 \mathrm{~mm}$, while the stone chips used as coarse aggregate for concrete was in the range of $4.75-19 \mathrm{~mm}$. The cement used in this study was a commercial ASTM type I ordinary Portland cement.

Table 1: Physical properties of waste plastic (HDPE) and poly bubble

\begin{tabular}{|l|l|l|}
\hline Type & HDPE & PUF \\
\hline Density (gm/cc) & 0.96 & 0.013 \\
\hline Water absorption & $0.10 \%$ & $0.20 \%$ \\
\hline Particle size & $4-12 \mathrm{~mm}$ & $4.0-8.5 \mathrm{~mm}$ \\
\hline
\end{tabular}

\subsection{Recycling of waste polymer}

The post consumer waste polymer materials had been collected, shredded and washed. Secondly, dried shredded materials were sieved. The particles of HDPE within the size-range of $4-12 \mathrm{~mm}$ were used as coarse aggregate. The particles of recycled PUF in the sizerange of 4.0-8.5 mm were used as the fine aggregate for the preparation of poly blocks and the particles with larger sizes than that were sent to the shredder for further size reduction.

\subsection{Preparation of concrete / block specimens}

For the preparation of concretes, cement: fine aggregates (sand): coarse aggregates (stone chips and HDPE) ratio of 1:2:4 in weight basis and water: cement ratio of $0.40: 1$ was used. The amounts of waste plastic (HDPE) added were 10\%, 15\%, 20\% and 25\% based on the weight of stone chips which are approximately equivalent to $10 \%, 15 \%, 20 \%$ and $25 \%$ of total volume of the sample. To prepare the fresh concrete, calculated amount of cement, sand, stone chips, waste plastic (HDPE) and water were mixed following a method and technique as prescribed by ASTM C 31-84. To examine the workability (or flow ability) of concrete, slump tests were done for the fresh concretes. The test procedure described in ASTM C 143-78 involved a cone shape mold, $203 \mathrm{~mm}$ in bottom diameter and $102 \mathrm{~mm}$ in top diameter. Then, the fresh concretes were cast into 152.4 $\mathrm{mm}$ cube molds with vibration following the procedure prescribed by ASTM, C31-84. The concrete specimens were separated out from the mold after 24 hours of molding and kept in water for 7-28 days for curing.

For the masonry poly blocks, cement: sand: PUF ratio was 1:0.5:1-8.5 on volume basis. The composition of PUF was equivalent to $33-90 \%$ of total volume of the sample. Water: cement ratio was kept constant at $0.40: 1$. Portland cement, sand, poly bubble and water were mixed together following a method and technique as prescribed by ASTM C 31-84 and were cast into a mold of size $240 \mathrm{~mm} \times 112 \mathrm{~mm} \times 112 \mathrm{~mm}$ to make masonry poly block.

\subsection{Methods}

For the characterization of the concrete/ masonry poly block, the compressive strength of the specimens was determined as a representative parameter of the mechanical properties, density and water absorption were determined as the representative parameters of physical properties, and porosity was determined as the representative parameters of morphological properties.

\subsubsection{Determination of water uptake of poly blocks}

The masonry poly block samples of size $120 \mathrm{~mm} \times 112 \mathrm{~mm} \times 112 \mathrm{~mm}$ were dried in an oven at 100 ${ }^{\circ} \mathrm{C}$ until a constant weight $w_{0}$ of the specimens was attained. Then the dried sample was immersed in water in a bowl at room temperature. At a predetermined time interval, the samples were taken out and the water adhered to the surfaced was wiped out by cloth and weighed. Then the sample was returned in the bowl. In the beginning, the measurement was done at an interval of 1 hour, and later on at large intervals. The equilibrium was reached in 48 hours. 
The water absorption, $A_{t}$, at a given time $t$ and the equilibrium water uptake, $A_{\infty}$, at $t \rightarrow \infty$ are calculated by the following formulae:

Where $w_{t}$ is the weight of the wet sample at time $t$ and $w_{\infty}$ is the weight at equilibrium.

The porosity $\beta$ of the poly blocks was calculated based on the water uptake data (Eqs. 2-4).

$$
\begin{aligned}
& \rho_{a}=w_{0} / V_{0} \\
& \rho_{T}=\frac{w_{0}}{V_{0}-\left(w_{\infty}-w_{0}\right) / \rho_{w}}
\end{aligned}
$$

and

$$
\beta=1-\rho_{a} / \rho_{T}
$$

Where $\rho_{a}$ and $\rho_{T}$ are the apparent and the true density respectively, $V_{0}$ is the bulk volume of the sample, and $\rho_{w}$ is the density of water.

\subsubsection{Measurement of compressive strength}

The compressive strength of different concrete and poly blocks specimens were determined using a Universal Testing Machine (Model No. TIB/ M. C; Capacity: 300 ton). The specific compressive strength of the material is defined as the compressive strength per unit specific gravity.

\section{Results \& Discussion}

The effect of the recycled PUF and HDPE on the specific compressive strength of the poly blocks and the concrete is shown in the Fig. 1.

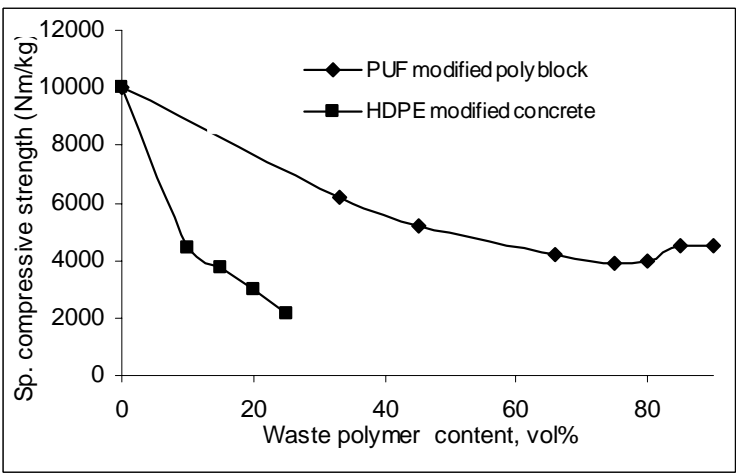

Fig. 1: Dependence of recycled polymer materials (PUF and HDPE) content on the compressive strength of the poly block and concrete

Fig. 1 shows that the specific compressive strength of HDPE-modified concrete decreases sharply with the increase in the HDPE content in the composition. In case of masonry poly blocks, the sp. strength decreases with the PUF content up to 75 vol\% of the sample, and shows slightly increasing trend beyond that. In fact, the

$$
A_{t}=w_{t} / w_{0}-1 \text { and } A_{\infty}=w_{\infty} / w_{0}-1
$$

addition of poly bubble results in the decrease in both the compressive strength and the density of poly blocks. The reduction of compressive strength of poly blocks might be due to either a poor bond between the cement paste and plastic aggregates or to the lower strength of the plastic aggregates.

It is found experimentally that the highest HDPE content that can be introduced in the concrete composition is $25 \mathrm{vol} \%$ and the highest introducible PUF content in poly blocks is 90 vol\%. More than these amounts in respective compositions creates problem in mixing and bonding, and hence the study is made up to the 25 vol\% of HDPE for concrete and 90 vol\% of poly bubble for poly blocks.

Kumar and Prakash $^{7}$ reported two times higher compressive strength using $5 \mathrm{wt} \%$ of waste plastic materials based on the total weight of the cement and the sand. To the contrary, Kumar et al. ${ }^{10}$ reported that the compressive, as well as tensile strength of concrete decreases with the use of waste plastics in concrete. Similarly, Al-Manaseer and Dalal ${ }^{4}$ found 34\%, 51\% and $67 \%$ reduction in compressive strength for concrete containing $10 \%, 30 \%$ and $50 \%$ plastic aggregates. Soroushian et al. ${ }^{5}$ also reported the reduction in compressive strength with the inclusion of recycled plastic.

Like most of the previous works, in this work also, the inclusion of waste polymer (both HDPE and PUF) is found to result in the decrease of compressive strength of concrete and masonry poly blocks (Fig.1). The rate of reduction in the compressive strength of the HDPE modified concrete is about $0.6 \mathrm{MPa}$ per volume percent of HDPE added while; the rate of reduction in compressive strength of poly blocks is only $0.21 \mathrm{MPa}$ per volume percent of Poly bubble added.

The density of the poly blocks decreases sharply with the PUF content as shown in the Fig. 2. The density of the poly blocks with $80 \mathrm{vol} \%$ of poly bubble content is low enough to make the blocks floatable in water. The density of HDPE- modified concrete also decreases with the increase in the HDPE content. But as HDPE is denser than the poly bubble, the HDPE modified concrete becomes heavier than the poly blocks. The reduction in the density of the poly blocks and the concrete is observed due to the lower unit weight property of the recycled plastic materials.

Al-Manaseer and Dalal ${ }^{4}$ reported that the bulk density of concrete was reduced $2.5 \%, 6 \%$ and $13 \%$ for the concrete containing $10 \%, 30 \%$ and $50 \%$ plastic 
aggregates respectively. The rate of reduction in the density of the HDPE-based concrete as observed in the present work was $0.2 \%$ per volume percent of waste polymer and that of the poly blocks is $0.77 \%$ per volume percentage of poly bubble content. This rate of reduction is higher than that reported by Al-Manaseer and Dalal ${ }^{4}$.

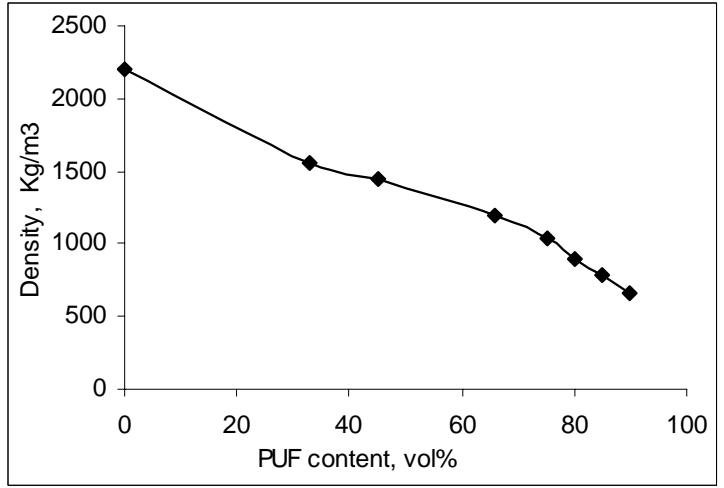

Fig. 2: Effect of PUF content on the density of the poly blocks

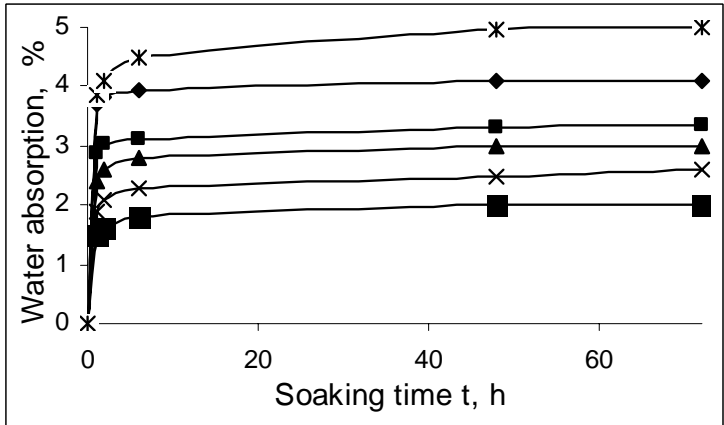

Fig. 3: Water absorption kinetics of poly blocks made of various PUF contents. Poly blocks modified with (*) 0 vol\%, (•) 33 vol\%, () 45 vol\% , (A) 66 vol\%, (×) 75 vol\% and (ם) 90 vol\% poly bubble

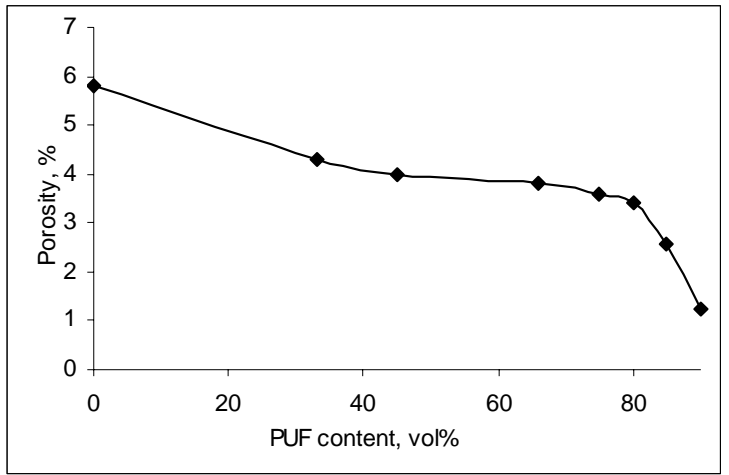

Fig. 4: Effect of PUF content on the porosity of the poly blocks
Fig. 3 shows the water absorption by poly blocks as a function of soaking time $t$ for various PUF contents. As seen from the Fig.3, initially the water absorption rate is very fast for all compositions of the poly blocks and then it gradually attains an equilibrium value. The water uptake value increases with the poly bubble content and is a combined effect of the porosity and the composition of the poly blocks material.

The Fig. 4 shows that the addition of PUF reduces the porosity of poly blocks. It can be explained by the hydrophobic nature of PUF. About 33 to 90 vol\% of the sample was occupied by the PUF which has low water absorption capacity and that result in the decrease in the porosity. The porosity decreases sharply at a concentration higher than $75 \mathrm{vol} \%$.

\section{Conclusion}

1) The incorporation of polyurethane formaldehyde into the masonry poly blocks makes the material very light compared to HDPE modified concrete. PUF based poly blocks can respond to the many needs of current and future construction works where light weight materials are recommended. The inclusion of recycled polymer materials however, decreases the compressive strength of the modified concrete and poly blocks. Moreover, due to have some exclusive properties such as sound and heat insulation, and energy efficiency of the waste polymer materials, concrete/poly blocks modified with waste polymer possesses remarkable potential to be utilized as construction materials in Bangladesh.

2) Inclusion of the PUF reduces water absorption and porosity of the poly blocks. The compressive strength of the materials also decreases with the poly bubble content in the composition.

\section{Acknowledgement}

This study was supported by the Housing and Building Research Institute. The authors would like to acknowledge the experimental facilities provided by the Chemical Testing and Research Laboratory (CTRL) and the Physical Testing and Research laboratory (PTRL) of HBRI.

\section{Reference}

1. United Nations Environment Program (2009), Converting waste Plastics into a resource, Industry and Economics International Environmental Technology Centre, Osaka/Shi, 1-69. 
2. Waste Concern Consultant (2006), Report on composition of plastic waste and market assessment of the plastic recycling sector in Dhaka city, 1-79.

3. Rebeiz, K.S., Serhal, S.P. and Fowler, D.W. (1994), Structural behavior of polymer concrete beams using recycled plastic, J. Mater. Civ. Eng. 6(1):150-165.

4. Al-Manaseer, A.A. and Dalal, T.R. (1997), Concrete containing plastic aggregates, J. Concr. Int. 19(8): 47-52

5. Soroushian, P., Plasencia, J. and Ravanbakhsh, S. (2003), Assessment of reinforcing effects of recycled plastic and paper in concrete, ACI Mater. J. 100(3):2003-2007

6. Elzafraney, M., Soroushian, P. and Deru, M. (2005), Development of energy-efficient concrete buildings using recycled plastic aggregates, $J$. Archit. Eng. 122-130

7. Kumar, K.B.V., and Prakash, P. (2006). Use of waste plastic in cement concrete pavement, $A d v$. Mater. Res. J. 15:1-21.
8. Ruzaidi, C.M., Norazian, M.N., Kamarudin, H. and Tarmizi, M. (2007), Strength of lightweight concrete with different percentage of modified polystyrene (MPS) aggregate, Proceedings of the International Conference on Sustainable Materials-ICoSM, Penang, Malaysia

9. Batayneh, M., Marie, I. and Asi, I. (2007), Use of selected waste materials in concrete mixes, $J$. Waste Manage. 27:1870-1876

10. Kumar, M., Dev, S.G. and Yadav, I.S. (2008), Laboratory Investigations of the properties of concrete containing recycled Plastic aggregates, M.Sc.Engg. thesis, Civil Engineering Department, Thapar University, Patiala, India.

11. Siddique, R., Khatib, J. and Kaur, I. (2008), Use of recycled plastic in concrete: A review, J. Waste Manage. 28:1835-1852 